\title{
Verification of the Universal Versatility of a Quantitative Protein Measurement Technique Using a Metal Mesh Device
}

\author{
Seiji Kamba, ${ }^{*}$ Hirokazu SETo, ${ }^{* *}$ Takashi Kondo, ${ }^{*}$ and Yoshiko MiUra***† \\ *Murata Manufacturing Co., Ltd., 1-10-1 Higashikotari, Nagaokakyo, Kyoto 617-8555, Japan \\ **Department of Chemical Engineering, Fukuoka University, 8-19-1 Nanakuma, Jonan, Fukuoka 814-0180, \\ Japan \\ ***Graduate School of Engineering, Kyushu University, 744 Motooka, Nishi, Fukuoka 819-0395, Japan
}

\begin{abstract}
When proteins are attached to microstructures such as a metal mesh device, changes in their optical properties occur. These changes have been characterized based on actual measurements in the infrared region of the spectrum. We have previously theoretically and experimentally demonstrated the optical changes associated with streptavidin. Here, we investigate three types of proteins: avidin, BSA, and lysozyme. The three proteins were adsorbed onto three types of metal mesh devices having different resonant frequencies, and the corresponding spectra were measured in the infrared region. The change in the frequency of the dip point in the spectrum was extracted to quantitatively determine the quantity of protein; these results were correlated with the quantitative measurements obtained by electrophoresis. By examining three types of different proteins, it was verified that a variety of proteins can be measured based on the optical characteristics of metal mesh devices.
\end{abstract}

Keywords Metal mesh device, SPR, quantitative measurement, avidin, BSA, lysozyme

(Received October 16, 2017; Accepted January 16, 2018; Published July 10, 2018)

\section{Introduction}

A metal mesh device (MMD) is a structure in which through holes of the same shape are intermittently arranged in a thin metal film. MMDs have optical characteristics corresponding to the periodic structure of the through holes. These optical characteristics include the electromagnetic wave transmission characteristics (as in a band-pass filter) through which electromagnetic waves in only a specific frequency band can pass. As the frequency of the transmission band is determined by the period of the pattern of through holes, the frequency of the passband can be controlled over a wide range from microwaves to the visible-light wavelengths by changing the MMD structure.

The electromagnetic wave transmission characteristics of an MMD is called extraordinary optical transmission. ${ }^{1}$ Previous research has been conducted with the aim of clarifying the cause of this mechanism. ${ }^{2,3}$ Further, these optical properties have been hardly used in industry and, band-pass filters in specialized fields, such as radio astronomy and high-frequency electromagnetic research, have been put to practical use, only recently., ${ }^{4,5}$

Among the early research utilizing the optical characters of MMDs, Miyamaru et al. in 2006 and Yoshida et al. in 2007 demonstrated the changes in the characteristics of electromagnetic wave transmission when a material is adsorbed onto an MMD due to changes in the optical properties. ${ }^{6,7}$ Additionally, the possibility to measure the mass of adsorbed

† To whom correspondence should be addressed.

E-mail: miuray@chem-eng.kyushu-u.ac.jp matter was demonstrated, which launched further studies about applications in sensors. Furthering the use of MMDs as sensing technologies, our research group reported the mechanism of resonance of MMDs in 2011 and demonstrated protein detection in $2013 .{ }^{8-11}$

In previous studies, it has been suggested that the electromagnetic wave transmission properties change as a result of protein adsorption onto the surface, but the basic characteristics of this change have not been sufficiently clarified. A correlation has been demonstrated between changes in the frequency [THz] spectrum of the electromagnetic wave transmission of MMD, caused by protein adsorption and the concentration $\left[\mathrm{nmol} \mathrm{mL}^{-1}\right]$ of the protein in the solution in which the MMD is immersed to adsorb protein. However, a quantitative investigation of this effect, which is a basic feature of an MMD-based sensor, remains insufficient.

To address this problem, our group reported in a previous article that the specific adsorption of streptavidin onto an MMD having biotin molecules immobilized on its surface was examined as a model. A proportional relation was identified between the frequency change in the electromagnetic wave transmission characteristics of the MMD upon adsorption of streptavidin $[\mathrm{THz}]$ and the mass of streptavidin $\left[\mathrm{ng} \mathrm{mm}^{-2}\right]$ adsorbed on the MMD surface as determined via electrophoresis. In addition, we demonstrated that by changing the structure of the MMD and changing the frequency of its passband, the sensitivity $\left[\mathrm{THz} \mathrm{ng} \mathrm{mm}^{-1} \mathrm{~mm}^{2}\right.$ increases in proportion to the square of the frequency of the MMD $\left[\mathrm{THz}^{2}\right] .{ }^{12}$ In the previous study, the possibility for quantitative measurement of streptavidin, a type of protein, using the MMD was demonstrated but the broad versatility that is necessary for practical application remained 


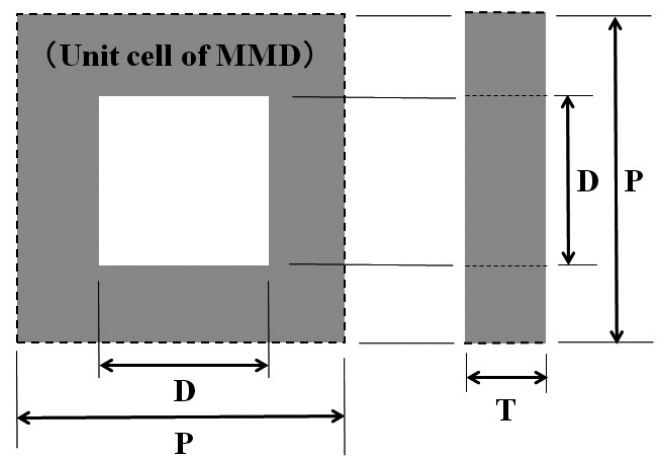

Fig. 1 Definitions of the structure parameters of MMD.

unproven. For practical applications, quantitative measurements regardless of the type of protein are ideal. Hence, it remained to be seen whether the same quantitative measurements could be realized for other proteins.

Therefore, in this study, we investigated the versatility of this quantitative protein-measurement technique with different types of proteins. Changes in the electromagnetic wave transmission characteristics when three proteins-avidin, BSA, and lysozyme - were adsorbed on the MMD were studied in detail. The quantity of attached protein was also measured independently by electrophoresis to study the relation between the amount of adsorbed protein and the resulting optical characteristics. Furthermore, three types of MMDs with different passband frequencies were prepared and the influence of the different light-absorption characteristics of proteins at each frequency on the sensing results was studied.

Surface plasmon resonance (SPR) in the visible light region, which operates on the same optical properties as MMDs, has been put to practical use as a technique for quantitative determination of small quantities of protein, particularly in the field of biopolymers. ${ }^{13}$ However, while SPR offers the advantage of accurate sensing, it is limited by the very small detectable light region from the sensor surface (100 nm or less). ${ }^{14}$ In addition, because the sensor chip is expensive, the chip must be recycled for reuse, which requires a complicated process. On the contrary, MMDs are manufactured using an electroforming method, enabling mass production of MMDs with various shapes. As the optical characteristics of the MMD can be controlled by varying the shape, it is possible to design an MMD with a detectable light region suitable for the size of the specific object to be measured. In addition, since MMDs can be produced at a low cost, they can be used as disposable sensing chips. Thus, this study aims to establish the broad applicability of MMDs as protein-sensing tools.

\section{Experimental}

\section{Experimental samples}

Figure 1 shows an example of the structure of an MMD and defines the structural parameters. In this study, three types of MMDs with different structures were prepared. These three types were selected based on the absorbance derived from the stretching vibration of the $\mathrm{OH}$ group, which occurs around $100 \mathrm{THz}$ in the absorption spectrum of a protein. The MMD sample name indicates that the frequency of the passband where electromagnetic wave transmission occurs, "f100", was designed to have a passband around $100 \mathrm{THz}$, which overlaps with the
Table 1 Structural parameters of three types of MMDs designed for this study

\begin{tabular}{cccc}
\hline \multirow{2}{*}{$\begin{array}{c}\text { Structural } \\
\text { parameter }\end{array}$} & \multicolumn{3}{c}{ Type of MMD } \\
\cline { 2 - 4 } & $\mathrm{f} 70$ & $\mathrm{f} 100$ & $\mathrm{f} 150$ \\
\hline$P / \mu \mathrm{m}$ & 3.6 & 2.6 & 1.7 \\
$D / \mu \mathrm{m}$ & 2.5 & 1.9 & 1.2 \\
$T / \mu \mathrm{m}$ & 1.0 & 0.8 & 0.6 \\
\hline
\end{tabular}

absorbance frequency associated with the $\mathrm{OH}$ group, and samples "f70" and "f150" were designed to have passbands around 70 and $150 \mathrm{THz}$, which do not overlap with the $\mathrm{OH}$ group absorbance frequency. Table 1 shows the structural parameters of each sample design: the period $(P)$, the hole size $(D)$, and the thickness $(T)$. The MMD samples were fabricated with an outer diameter of $6 \mathrm{~mm}$ from $\mathrm{Ni}$ via electroforming. Then, electroless plating was used to apply a layer of Au several tens of nanometers in thickness entirely on the electroformed $\mathrm{Ni}$ MMD before use for test samples.

Three types of protein were tested in this study: avidin (Wako, 011-24233), bovine serum albumin (BSA) (Wako, 014-15134), and lysozyme (Wako, 120-05011).

\section{Absorbance measurements of the proteins}

To compare the basic optical characteristics of the three proteins, their absorption spectra were measured in the range from the infrared region to the visible region using an FT-IR instrument (Bruker Optics, Vertex-70V) and a UV-VIS device (Hitachi High-Tech Science Co., U-4100).

In the infrared region, the measurements were performed using the FT-IR apparatus with the $\mathrm{KBr}$ method. $\mathrm{KBr}$ powder and protein powder were mixed and pelletized to contain a protein content of approximately $1 \mathrm{wt} \%$. The transmittance of the pellet was then measured to obtain the absorption spectrum of the protein. To measure the absorbance in the visible light region, a UV-VIS apparatus was used with an aqueous solution of the protein adjusted to have the same amount of protein as the pellet used in the $\mathrm{KBr}$ method. Drops of the sample were dried on a glass slide to obtain a thin film of the protein from which the absorption spectrum was measured.

Protein adsorption to the MMD and electromagnetic wave transmission measurements

Nonspecific adsorption to the surfaces of three types of MMDs was tested with the three types of proteins according to the following procedure. Three solutions were prepared in PBS: $1 \mathrm{mg} \mathrm{mL}{ }^{-1}$ avidin, $10 \mathrm{mg} \mathrm{mL}^{-1} \mathrm{BSA}$, and $3 \mathrm{mg} \mathrm{mL}^{-1}$ lysozyme. One piece of MMD was immersed in $1 \mathrm{~mL}$ of a protein solution and incubated at $37^{\circ} \mathrm{C}$ for $2 \mathrm{~h}$ to allow adsorption. Then, the MMD was washed with PBS and ultrapure water and subsequently dried in vacuo.

The electromagnetic wave transmission measurements of each MMD were performed using an FT-IR device (Bruker Optics, ALPHA) before and after protein adsorption. Based on the difference between the two absorption spectra, the change in electromagnetic wave transmission characteristics due to protein adsorption was determined. On each absorption spectrum, the bottom point in the passband of the waveform where the transmittance fell sharply was defined as the dip point and the difference between the frequencies at which the dip point was observed before and after protein adsorption was quantified. For each experimental condition, eight MMD measurements 


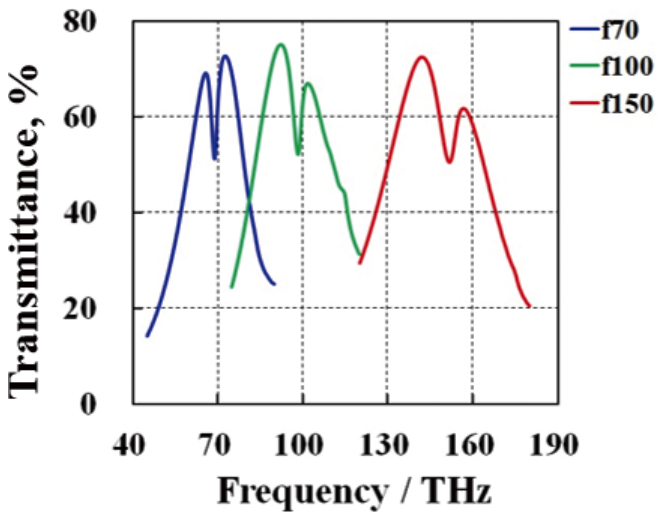

Fig. 2 Electromagnetic wave transmission characteristics in the infrared region of the MMD samples: f70, f100, and f150.

were made and the average of these measurements was taken as the measured value.

\section{Determination of protein mass by electrophoresis}

The mass of the protein adsorbed on the MMD was determined via electrophoresis in the same manner as previously reported. ${ }^{12}$ In this procedure, after measuring the electromagnetic wave transmission of an MMD following protein adsorption, the MMD was immersed in a reagent solution and heated to remove the adsorbed proteins from the MMD into the solution. Electrophoresis was then conducted on this solution and protein solutions of known concentration as references for the calibration curve. The protein bands were stained with fluorophores for visualization, and the obtained band images were analyzed using "Image-J," a free software, to determine the mass of the protein adsorbed on the MMD. For each experimental condition, electrophoresis was repeated in nine lanes and the average of the resulting masses was taken as the measured value.

\section{Results}

Electromagnetic wave transmission characteristics of MMD

For the three types of MMDs- "f70," "f100," and "f150"the electromagnetic wave transmission characteristics before adsorption of protein were measured by FT-IR. The results are shown in Fig. 2 with the frequency [THz] on the horizontal axis and the transmittance [\%] on the vertical axis; the blue, green, and red lines show the spectra for the "f70," "f100," and "f150" MMD samples, respectively. According to these results, "f70" had a passband around $70 \mathrm{THz}$ and the frequency of the dip point in the passband was approximately $68.5 \mathrm{THz}$; "f 100 " had a passband around $100 \mathrm{THz}$ and the frequency of the dip point was approximately $98.4 \mathrm{THz}$; and "f150" had a passband around $150 \mathrm{THz}$ and the frequency of the dip point was approximately 151.5 THz.

\section{Absorption spectra of proteins in the infrared region}

The absorption spectra in the infrared region were measured for the three proteins (avidin, BSA, and lysozyme) using the $\mathrm{KBr}$ method. The results are shown in Fig. 3, with the horizontal axis representing the frequency $[\mathrm{THz}]$ and the vertical axis representing the absorbance [arbitrary unit] normalized to the magnitude of the absorption peak near $100 \mathrm{THz}$; the blue, red, and green lines represent the spectra for avidin, BSA, and lysozyme, respectively. These results demonstrate that the

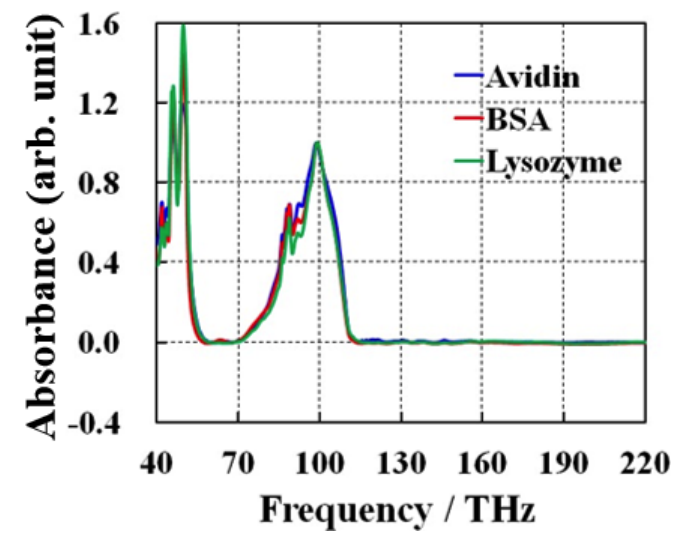

Fig. 3 Measured absorbance spectra of the proteins in the infrared region.

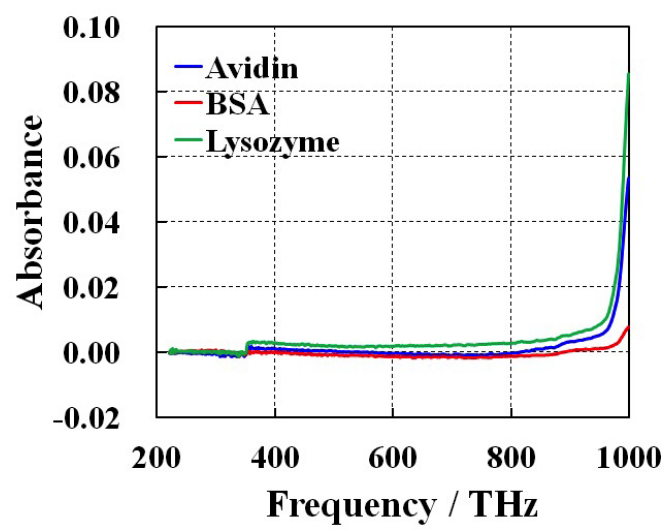

Fig. 4 Measured absorbance of the proteins in the visible region.

absorption spectra of the three proteins are consistent with each other in the frequency range of $40-220 \mathrm{THz}$ (corresponding to wave numbers in the range of $1333-7333 \mathrm{~cm}^{-1}$ ) within the infrared range measured here. Furthermore, the absorbance of each of the three proteins was measured. In the case of $\mathrm{f} 70$ and f150, there was no absorbance at the dip point frequency (average absorbance of 0,000 ), and in the case of f100, the absorbance of proteins at the dip point frequency was observed. The relative absorbance of proteins at $\mathrm{f} 100$ dip frequency was much larger than that at $\mathrm{f} 70$ and $\mathrm{f} 150$.

These results demonstrate that various types of MMDs can be fabricated with dip point frequencies based on the absorption band associated with the stretching vibration of the $\mathrm{OH}$ groups of proteins around $100 \mathrm{THz}$.

\section{Absorption spectra of proteins in the visible region}

The absorption spectra of the three proteins were also measured in the visible region. The results are shown in Fig. 4, wherein the horizontal and vertical axes represent the frequency $[\mathrm{THz}]$ and the absorbance, respectively, and the blue, red, and green lines correspond to the spectra of avidin, BSA, and lysozyme. These results indicate that the absorption spectra of the three proteins are almost coincident in the frequency range of $222-1000 \mathrm{THz}$ (corresponding to wavelengths in the range of $1350-300 \mathrm{~nm}$ ). Further, the absorbance was almost zero across the whole frequency range except for the increase in 
Table 2 Experimentally measured $\Delta f$ values due to different amounts of adsorbed protein, $M$

\begin{tabular}{|c|c|c|c|c|c|c|}
\hline \multirow{3}{*}{$\begin{array}{l}\text { Type of } \\
\text { MMD }\end{array}$} & \multirow{3}{*}{$\begin{array}{l}\text { Type of } \\
\text { protein }\end{array}$} & \multicolumn{5}{|c|}{ Measurement result } \\
\hline & & \multicolumn{2}{|c|}{$\Delta f / \mathrm{THz}$} & \multicolumn{2}{|c|}{$M / \mathrm{ng} \mathrm{mm}^{-2}$} & \multirow{2}{*}{$\begin{array}{c}A / \mathrm{THz} \\
\mathrm{ng}^{-1} \mathrm{~mm}^{2}\end{array}$} \\
\hline & & Ave. & $\sigma$ & Ave. & $\sigma$ & \\
\hline f70 & Avidin & 0.074 & 0.021 & 3.11 & 0.56 & 0.024 \\
\hline f70 & BSA & 0.071 & 0.026 & 3.15 & 0.25 & 0.022 \\
\hline f70 & Lysozyme & 0.148 & 0.013 & 4.61 & 0.77 & 0.032 \\
\hline f100 & Avidin & 0.142 & 0.023 & 3.35 & 0.95 & 0.042 \\
\hline $\mathrm{f} 100$ & BSA & 0.245 & 0.039 & 4.12 & 0.34 & 0.059 \\
\hline $\mathrm{f} 100$ & Lysozyme & 0.259 & 0.019 & 4.63 & 1.04 & 0.056 \\
\hline $\mathrm{f} 150$ & Avidin & 0.355 & 0.081 & 3.56 & 0.81 & 0.100 \\
\hline $\mathrm{f} 150$ & BSA & 0.477 & 0.117 & 4.67 & 0.37 & 0.102 \\
\hline f150 & Lysozyme & 0.475 & 0.068 & 4.44 & 0.49 & 0.107 \\
\hline
\end{tabular}

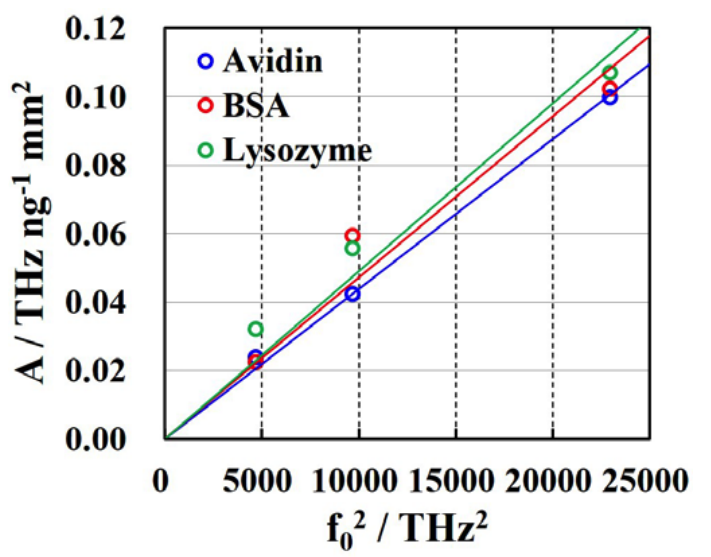

Fig. 5 Relation between the frequency, $f_{0}$, at the dip point and the sensitivity, $A$.

absorbance around $1000 \mathrm{THz}$. Note that the discontinuities in the spectra near $350 \mathrm{THz}$ are artifacts from the device and are known to be independent of the absorbance of the protein.

Amount of adsorbed protein and change in frequency of the dip point due to protein adsorption

The change in the frequency of the MMD dip point, $\Delta f[\mathrm{THz}]$, due to nonspecific adsorption of each of the three types of proteins (avidin, BSA, and lysozyme) on each of the three types of MMDs (f70, f100, and f150) was measured by FT-IR. The mass, $M\left[\mathrm{ng} \mathrm{mm}^{-2}\right]$, of the protein adsorbed on the MMD surface was then quantified via electrophoresis. Table 2 shows the measured $\Delta f$ and $M$ per unit area of each MMD with each protein as well as the sensitivity, $A\left[\mathrm{THz} \mathrm{ng}{ }^{-1} \mathrm{~mm}^{2}\right]$, calculated as $\Delta f / \mathrm{M}$. These results indicate that the sensitivity of the "f70", "f100", and "f150" MMDs were approximately 0.02, 0.05, and 0.10 , respectively, regardless of the type of protein. Figure S1 (Supporting Information) shows an example of a gel image with fluorescent staining used for the quantitative determination of avidin. The history of each lane of the gel image in Fig. S1 (Supporting Information) is shown in Table S1 (Supporting Information) of the supporting data.

Relation between the dip point frequency of the MMD and the sensitivity of protein sensing

The relation between the frequency, $f_{0}[\mathrm{THz}]$, of the dip point (shown in Fig. 2) and the sensitivity, $A$ [THz ng-1 $\left.\mathrm{mm}^{2}\right]$ (shown in Table 2), is shown in Fig. 5 for "f70", "f100", and "f150" MMDs. The horizontal axis in the figure is the square of the frequency of the dip point, $f_{0}^{2}\left[\mathrm{THz}^{2}\right]$, and the vertical axis is the sensitivity, $A\left[\mathrm{THz} \mathrm{ng}^{-1} \mathrm{~mm}^{2}\right]$. The blue, red and green plotted points shown the results obtained with avidin, BSA, and lysozyme, respectively, and the straight lines show the corresponding linear regression lines calculated from this data. Based on these results, the square of the frequency of the dip point, $f_{0}^{2}$, is proportional to the sensitivity, $A$, regardless of the type of protein and that the proportionality constants were similar: $4.4 \times 10^{-6}\left[\mathrm{THz}^{-1} \mathrm{ng}^{-1} \mathrm{~mm}^{2}\right]$ for avidin, $4.7 \times 10^{-6}$ $\left[\mathrm{THz}^{-1} \mathrm{ng}^{-1} \mathrm{~mm}^{2}\right]$ for BSA, and $4.9 \times 10^{-6}\left[\mathrm{THz}^{-1} \mathrm{ng}^{-1} \mathrm{~mm}^{2}\right]$ for lysozyme.

\section{Discussion}

\section{Summary of experimental results}

The three types of proteins tested here are very different biopolymers; the amino acid sequence and the types of amino acids in the chains have no relation with each other. However, they have similar spectra in both the infrared and the visible light regions.

Table S2 (Supporting Information) lists the dielectric constants of all amino acids in the $\mathrm{THz}$ band, as measured using $\mathrm{THz}$ time-domain spectroscopy (THz-TDS). This data indicates that, although the dielectric constants of the 20 types of amino acids vary, the difference between them is small and falls within a certain range. This suggests that proteins, which can be thought of as aggregates of amino acids, have similar optical properties.

From these results, it was concluded that the relation between $\Delta f$ and $M$ is similar for the three types of proteins.

\section{Similarity between the mechanisms of protein quantification by $S P R$ and $M M D$}

SPR operates based on the plasma oscillation of metal and the resonance phenomenon of electromagnetic waves. When a protein enters the electromagnetic resonant field formed by the electromagnetic waves near the metal surface, the refractive index in the space of the resonance electromagnetic field changes, leading to a change in the resonance state. This change can be observed as a change in the resonance angle, $\omega$, when a protein is adsorbed on the metal surface. In contrast, MMD operates based on the resonance phenomenon of an MMD structure and electromagnetic waves: when a protein enters the electromagnetic resonant field formed by the electromagnetic waves near the MMD, the dielectric constant (i.e., the refractive index) of the resonant electromagnetic field space changes; this change causes a change in the resonance state, which can be detected as a change in the frequency, $\Delta f[\mathrm{THz}]$, of the dip point when a protein is adsorbed on the metal surface. SPR and MMD are similar in that they are both based on the changes in their resonance state, but the electromagnetic wave resonance is caused by different mechanisms.

In SPR, it is assumed the amount of protein adsorption on the substrate is propotional to the resonance angle of $\omega$, where 0.1 degree shift corresponds to to $1 \mathrm{ng} \mathrm{mm}^{-2}$ of protein. ${ }^{15}$ Considering the principle of measurement of SPR described above, the refractive indices should change in the same way regardless of the type of protein adsorbed. If this premise did not hold, assuming the same amount of two adsorbed proteins, a protein with the larger refractive index would cause a very large change in $\omega$ while a protein with a small refractive index would cause a small change in $\omega$. Thus, the proportion of 


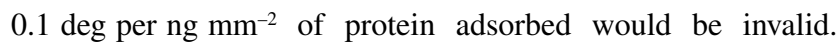
Generally, in SPR, visible light with a frequency of about $375-500 \mathrm{THz}$ (or a wavelength of $600-800 \mathrm{~nm}$ ) is used as the electromagnetic wave. As shown in Fig. 4, this frequency range is between the infrared region where absorbance occurs due to molecular vibration and the ultraviolet region where the absorption occurs due to electron excitation; as proteins generally have no absorption factor, the absorbance is almost zero. Thus, the above approximation was presumably established on the premise of proteins having identical optical properties in the visible region.

We considered the possibility of identifying an approximation for protein quantification based on an MMD similar to that used in SPR based on the similarities in their measurement principles. In the case of MMDs resonating in the infrared region as shown in Fig. 3, the absorption spectra of all proteins in the infrared region are similar. Therefore, assuming that the proteins have identical optical properties in the infrared region, it seems plausible to identify an approximation for MMD in the infrared region, similar to that of SPR in the visible region.

The optical properties of an MMD are dependent on the specific structure of the MMD, but it is reported that the phenomena has some similarity to SPR. Pendry reported that two dimensional metal photic crystals showed the SPR mimicking property with micro-level structure. ${ }^{16}$ Since an MMD is one of the metal photonic crystals, the similarity of SPR is suggested. We considered the detailed properties of MMDs need more investigation.

Versatility of the quantitative measurement of proteins using $M M D$

Three different proteins (avidin, BSA, and lysozyme) were adsorbed onto each of the three types of MMDs having different resonance frequencies (f70, f100, and f150) and the change in the frequency, $\Delta f$, at the dip point was measured as a function of the amount of protein adsorbed, $M$. The sensitivity, $A$, derived from the experimental data, as shown in Table 2, was consistent for different proteins with the same type of MMD. This finding suggests that the approximation for the protein amount that is used for SPR in the visible range is similarly valid for MMDs in the infrared region: $A\left[\mathrm{THz} \mathrm{ng}^{-1} \mathrm{~mm}^{2}\right]$ corresponds to the proportion used to determine the amount of protein adsorbed [ng $\mathrm{mm}^{-2}$ ] from the change in the resonance angle, $\omega$ [deg]. Based on these results, it was concluded that as in the case of SPR, regardless of the type of protein, the amount of protein adsorbed can be quantitatively determined using an MMD.

In the previous study, ${ }^{12}$ it was reported that the protein detection sensitivity, $A$, of the MMD increases in proportion to the square of the resonance frequency, $f_{0}$, of the MMD. Figure 5 shows that the resonance frequency, $f_{0}$, of the dip points for the three types of MMDs (f70, f100, and f150) is related to the sensitivity, $A$ : the value of $A$ increases in proportion to the square of the resonance frequency, $f_{0}$, as described in the previous study. The data also shows that this relation is substantially the same regardless of the type of protein. This relation can be described by averaging the three graphs in Fig. 5: $A=4.7 \times 10^{-6} \times f_{0}{ }^{2}$. Using this universal formula for the quantitative determination of proteins in an MMD, the sensitivity, $A$, can be obtained by measuring the resonance frequency, $f_{0}$, suggesting that it may be possible to quantify proteins at any frequency, as in SPR.

Influence of the resonance frequency of the $M M D$ on the quantitative detection of proteins

As shown in Fig. 3, at the frequencies of $\mathrm{f} 70$ and $\mathrm{f150}$, there is almost no absorption by the protein but the absorbance is large at a frequency of f100. Furthermore, as shown in Fig. 4, there is approximately zero absorbance by the protein in the visible region used for SPR (frequencies of approximately $350-500 \mathrm{THz}$ ). Since the absorbance of protein was not observed at the dip point frequency of 170 and $\mathrm{f} 150$, the optical property at the dip point seemed to be similar, though the refractive index is slightly different. The protein detection with f70 and f150 is not strongly affected by the protein refractive index like SPR biosensor. On the other hand, the protein detection with $\mathrm{f} 100$ has the possibility to be affected by protein absorbance, which is different from the protein detection with f70, f150 and SPR. The optical characteristics of the protein differ in the frequency range of $\mathrm{f} 100$ as proteins exhibit a large absorption. In this context, we examined how the differences in the absorbance characteristics of proteins affect their quantitative determination using an MMD.

In general, an increase in absorbance indicates either an increase in the reflection or absorption of the electromagnetic waves or a decrease in the transmission. In addition, an increase in reflection corresponds to an increase in the real part of the dielectric constant of the protein and an increase in absorption corresponds to an increase in the imaginary part. Changes in the absorption and reflection of the electromagnetic waves by the protein in the infrared region is derived from the molecular vibration and the resulting dielectric relaxation, which causes a frequency change in the real and imaginary parts of the dielectric constant. For example, Fig. S2 (Supporting Information) shows the frequency dispersion of the dielectric constant of an amino acid, DL-alanine, in the $\mathrm{THz}$ band as measured by THz-TDS. Although DL-alanine has an absorption peak at $1.25 \mathrm{THz}$, the data shows that both the relative dielectric constant, $\varepsilon_{\mathrm{r}}$, (corresponding to the real part of the dielectric constant) indicated by the blue line and the dielectric loss tangent, $\tan \delta$, (corresponding to the imaginary part) indicated by the red line significantly vary as a function of the frequency.

As shown in the previous study, ${ }^{12}$ the frequency change of the dip point due to protein adsorption on the MMD is related to the real part of the dielectric constant of the protein: as the real part of the dielectric constant increases, the change in frequency per unit of protein increases. The fact that the absorbance of the protein increases at the frequency of f100 indicates an increase in either the real or imaginary part of the dielectric constant of the protein at that frequency. This is attributable to the increase in the real part of the dielectric constant at the frequency of f100 compared to those at the frequencies of $\mathrm{f} 70$ or $\mathrm{f} 150$, at which there is almost no absorption. However, it should be noted that the change in the frequency at the dip point should be larger based on the increase in the real part of the dielectric constant. Figure 5 shows that the sensitivity, $A$, increases in proportion to the square of the frequency, $f_{0}$, of the MMD. As reported in the previous study, this relation holds when the real part of the dielectric constant of the protein is constant at all frequencies. On the contrary, if the real part of the dielectric constant of the protein at the frequency of f100 is larger than those at the frequencies of $\mathrm{f} 70$ or $\mathrm{f150}$, the graph in Fig. 5 would not be a straight line but would instead take the shape of an upwardly convex curve at the frequency of f100. However, the relation shown in Fig. 5 is linear, even at the frequency of $\mathrm{f} 100$ where a large absorption occurs; therefore, it is inferred that the absorption of proteins at the frequency of $\mathrm{f} 100$ is dominated by the absorption of the electromagnetic waves due to an increase in the imaginary part of the dielectric constant and that the increase in the real part of the dielectric constant is too small to affect the protein detection by the MMD. 
The spectra of MMD are changed based on the imaginary part of the dielectric constants of the proteins. Since the thickness of the proteins on the MMD is very thin and less than $100 \mathrm{~nm}$, the volume of the target substances (proteins) in TE11-like mode resonant space is too small to affect the dielectric constant change on the spectra of the dip point and the transmission. The computer calculation with different dielectric dissipation factor is shown in Fig. S3 (Supporting Information), where the dip point was almost the same. The real spectra of $f 100$ before and after lysozyme adsorption is shown in Fig. S4, which also shows the changes were not large. Therefore, though the absorbance of proteins around the dip point of $\mathrm{f} 100$ was different from $\mathrm{f} 70$ and $\mathrm{f} 150$, the sensitivity of the protein detection at $\mathrm{f} 100$ was similar

These results suggested that the protein detection with MMD is not strongly affected by the protein absorbance.

\section{Conclusions}

In this study, three different types of proteins were adsorbed onto three types of MMDs having different resonance frequencies and the resulting changes in the resonance frequency were measured. The experimental results demonstrated that the detection sensitivity for the amount of adsorbed protein and the change in the resonance frequency are proportional to the square of the resonance frequency. It was also observed that this relation was not significantly influenced by the absorbance of the protein itself. There is a possibility that their relation is not significantly affected, regardless of the type of the protein.

These results, combined with the noted similarity between the measurement principles of MMD and SPR, suggest that the approximation used for the quantitative measurement of protein by SPR is similarly applicable to protein measurements using MMDs. Thus, we predict that the quantitative determination of proteins using MMDs may be achieved with large versatility.

\section{Supporting Information}

Results of electrophoresis and measurement results of amino acid by THz-TDS. This material is available free of charge on the Web at http://www.jsac.or.jp/analsci/.

\section{References}

1. T. Ebbesen, H. Lezec, H. Ghaemi, T. Thio, and P. Wolff, Nature, 1998, 391, 667.

2. L. Martín-Moreno, F. J. García-Vidal, H. J. Lezec, K. M. Pellerin, T. Thio, J. B. Pendry, and T. W. Ebbesen, Phys. Rev. Lett., 2001, 86, 1114.

3. S. Nashima and Y. Ogawa, IEEJ Trans. EIS, 2013, 133, 484.

4. A. Mitsuishi, Y. Otsuka, S. Fujita, and H. Yoshinaga, Jpn. J. Appl. Phys., 1963, 2, 574.

5. R. Rawcliffe and C. Randa, Appl. Opt., 1967, 6, 1353.

6. F. Miyamaru, S. Hayashi, C. Otani, K. Kawase, Y. Ogawa, H. Yoshida, and E. Kato, Opt. Lett. 2006, 31, 1118.

7. H. Yoshida, Y. Ogawa, Y. Kawai, S. Hayashi, A. Hayashi, C. Otani, E. Kato, F. Miyamaru, and K. Kawase, Appl. Phys. Lett., 2007, 91, 253901.

8. T. Kondo, S. Kamba, K. Takigawa, T. Suzuki, Y. Ogawa, and N. Kondo, in Proceedings of EurosensorsXXV, ed. G. Kaltsas and C. Tsamis, 2011, 916.

9. H. Seto, C. Yamashita, S. Kamba, T. Kondo, M. Hasegawa, M. Matsuno, Y. Ogawa, Y. Hoshino, and Y. Miura, Langmuir, 2013, 29, 9457.

10. H. Seto, S. Kamba, T. Kondo, M. Hasegawa, S. Nashima, Y. Ebara, Y. Ogawa, Y. Hoshino, and Y. Miura, ACS Appl. Mater. Interfaces, 2014, 6, 13234.

11. H. Seto, S. Kamba, T. Kondo, Y. Ogawa, Y. Hoshino, and Y. Miura, Anal. Sci., 2015, 31, 173.

12. S. Kamba, H. Seto, T. Kondo, and Y. Miura, Anal. Sci., 2017, 33, 1033

13. J. Homola, J. Anal. Bioanal. Chem., 2003, 377, 528.

14. B. Liedberg, C. Nylander, and I. Lunström, Sens. Actuators, 1983, 4, 299.

15. E. Stenberg, B. Persson, H. Roos, and C. Urbaniczky, J. Colloid Interface, 1991, 143, 513.

16. J. Pendry, L. Moreno, and F. J. G. Vidal, Science, 2004, $305,847$. 